\title{
Targeting the gut microbiome for non-communicable diseases: present and future
}

\author{
Subhashree Shivani ${ }^{1}$, Amrita Chattopadhyay ${ }^{2}$, Eric Y. Chuang ${ }^{1,2,3}$ \\ ${ }^{1}$ Graduate Institute of Biomedical Electronics and Bioinformatics, National Taiwan University, Taipei; ${ }^{2}$ Bioinformatics and Biostatistics Core, Centre \\ of Genomic and Precision Medicine, National Taiwan University, Taipei; ${ }^{3}$ Biomedical Technology and Device Research Laboratories, Industrial \\ Technology Research Institute, Hsinchu \\ Correspondence to: Eric Y. Chuang. Department of Electrical Engineering, Graduate Institute of Biomedical Electronics and Bioinformatics, National \\ Taiwan University, Taipei 10617. Email: chuangey@ntu.edu.tw.
}

Submitted Jan 12, 2021. Accepted for publication Feb 07, 2021.

doi: 10.21037/atm-21-144

View this article at: http://dx.doi.org/10.21037/atm-21-144

In the $19^{\text {th }}$ century, Dr. William Coley (the father of immunotherapy) mixed streptococcal bacteria with medicine and successfully treated a patient with inoperable sarcoma (1). The idea was to stimulate an immune response due to the bacterial infection, which presumably helped in treating the cancer. Although chemotherapy and radiotherapy eclipsed this field of research, their adverse effects have rekindled research into bacterial therapy. The human body is inhabited by trillions of symbiotic bacteria and microbes that coevolved with human beings. Bacterial metabolites and bacteria-host interactions shape the biological well-being of the host by actively impacting multiple host functions. One of the important consequential abodes of bacteria is the gut. Thanks to the rapidly growing sequencing industry, scientists are extensively studying the composition of gut microbiota in relation to various demographic and clinical characteristics such as geography (2), sex (3), age (4), dietary habits (5), and body mass index (6).

Many studies have revealed the susceptibility of gut microbiota to a host's diseases $(7,8)$. Some studies have also shown the role of gut microbiota in modulating host response to immune checkpoint inhibitor cancer immunotherapy (9). Determination of healthy gut microbiota baselines is beneficial in tracking dysbiosis in many chronic non-communicable diseases such as Alzheimer's (10), kidney disease (11) and schizophrenia (12). As the microbiome plays an integral part in the metabolism of its host, recognizing the effect of a dysbiotic microbiome in diseases could help in providing alternate therapy. Gut microbiota can directly modulate coronary artery disease by producing bile acids, coprostanol, short chain fatty acids, and trimethylamine-N-oxide, or indirectly by manipulating the immune system (13). In cardiovascular disease (CVD), heart failure has been associated with specific gut microbial species like Escherchia coli, Klebsiella pneumoniae and Streptococcus viridian (14). In another study, author showed altered gut microbiota in patients with symptomatic strokes and transient ischemic attack with increased abundance of Enterobacter, Megasphaera, Oscillibacter and Desulfovibrio (15). In the case of chronic kidney disease, a dysbiotic gut microbiome produces excess uremic toxins such as phenols and indoles, which can't be completely removed during dialysis and lead to further complications (16). Wilkins et al. identified Bacteroides, Corynebacterium, Anaerococcus, Prevotella, Rothia, Sutterella, Eubacterium, Fusobacterium, Leptotrichia, Parabacteroides, Peptoniphilus, Porphyromonas, and Veillonella bacteria as the major genera associated with kidney diseases (17). Shen et al. compared the gut microbiota of 64 schizophrenic patients with 53 healthy individuals, aiming to identify potential biomarkers for schizophrenia (18). They identified changes in Gammaproteobacteria (classlevel), Enterobacteriales (order-level) and Bacteroides fragilis (species-level) with potential association with schizophrenia. Zheng et al. demonstrated that germ-free mice receiving a fecal microbiome transplant from a schizophrenia patient exhibited lower glutamate and higher glutamine and GABA in the hippocampus and displayed schizophrenialike behavior (19). They also identified a panel of Aerococcaceae, Bifidobacteriaceae, Brucellaceae, Pasteurellaceae, and Rikenellaceae bacteria with the capability to distinguish 
schizophrenia patients from healthy controls. Alzheimer's disease involves deposition of amyloid beta (A $\beta$ ) in the brain followed by formation of plaques and neurofibrillary tangles composed of hyperphosphorylated tau protein (20). These amyloid deposits are responsible for neuroinflammation leading to synapse loss and neuronal death (21). Gut Escherichia coli is a source of curli, a bacterial amyloid (22), and it has been shown that rats exposed to curli-producing $E$. coli displayed increased neuronal alpha-synuclein deposition in both the gut and brain, and enhanced microgliosis and astrogliosis compared to rats exposed to bacteria unable to produce curli (23).

Identifying the gut microbiota through 16 srRNA sequencing is the first step in microbial therapy. Considering the role of gut microbiota in pathways pertaining to multiple diseases, researchers are targeting the involved gut microbiota for potential therapy. Wang et al. showed how a gut microbiota imbalance facilitates infiltration of the brain by peripheral immune cells, contributing to cognitive impairment by enhancing microglial activation (24). GV971, a sodium oligomannate that has shown cognitive improvement in phase 3 trials in China, reverses cognitive impairment by suppressing gut dysbiosis and associated phenylalanine/isoleucine accumulation.

In another study, targeting the gut microbiome for kidney diseases, Devlin et al. described a novel approach to reduce the production of indoxyl sulfate (25). Using computational methods, the authors identified a tryptophanase gene present in some bacteria species colonizing the gut. Tryptophanase helps produce indole, a precursor to indoxyl sulfate, using tryptophan. Upon colonizing germfree mice with mutant bacteria harboring the deleted tryptophanase gene, authors observed no detectable serum or urinary indoxyl sulfate in the germ-free mice, in contrast to mice with wild-type bacteria. To determine if dietary intervention could alter the relative abundance of indoleproducing species, the authors colonized mice with both indole producer (wild-type Bacteroides theta) and nonindole producer (wild-type B. caccae) bacteria. A diet rich in fructo-oligosaccharides (favors growth of $B$. caccae) shifted the bacterial community structure and decreased urinary indoxyl sulfate levels.

Gut microbiota have emerged as a powerful alternative for therapy for complex diseases. Modulating the gut microbiota has efficiently suppressed disease complications in mouse models. Although this field is gaining momentum rapidly, this field of research is still in its infancy. The major challenge is confirming the dysbiosis of the gut microbiome as a cause or consequence of the disease. Also, along with gut microbiota, other host factors such as genetics and geography might play an important role in the efficacy of microbial therapy. Understanding the interactions between different bacteria in the gut and in the diseased sites is also important to optimize therapeutic conditions. In conclusion, understanding the complex interactions of targeted bacteria with their surroundings and the host is required to optimize the overall impact of bacterial therapy in the host and minimize side effects. Whole genome sequencing and a multi-omics approach is needed to unveil bacterial structure and mechanisms and broaden our understanding of hostbacteria interactions. Resolving these challenges will bring the use of bacterial therapy to its maximum potential and possibly initiate a new era of truly personalized medicines.

\section{Acknowledgments}

We thank Melissa Stauffer, Ph.D. for editing the manuscript.

Funding: This work was supported by Center of Biotechnology, National Taiwan University [GTZ300].

\section{Footnote}

Provenance and Peer Review: This article was a standard submission to the journal. The article did not undergo external peer review.

Conflicts of Interest: All authors have completed the ICMJE uniform disclosure form (available at http://dx.doi. org/10.21037/atm-21-144). The authors have no conflicts of interest to declare.

Ethical Statement: The authors are accountable for all aspects of the work in ensuring that questions related to the accuracy or integrity of any part of the work are appropriately investigated and resolved.

Open Access Statement: This is an Open Access article distributed in accordance with the Creative Commons Attribution-NonCommercial-NoDerivs 4.0 International License (CC BY-NC-ND 4.0), which permits the noncommercial replication and distribution of the article with the strict proviso that no changes or edits are made and the original work is properly cited (including links to both the formal publication through the relevant DOI and the license). See: https://creativecommons.org/licenses/by-nc-nd/4.0/. 


\section{References}

1. McCarthy EF. The Toxins of William B. Coley and the Treatment of Bone and Soft-Tissue Sarcomas. Iowa Orthop J 2006;26:154-8.

2. Yatsunenko T, Rey FE, Manary MJ, et al. Human gut microbiome viewed across age and geography. Nature 2012;486:222-7.

3. Kim YS, Unno T, Kim BY, et al. Sex Differences in Gut Microbiota. World J Mens Health 2020;38:48-60.

4. Odamaki T, Kato K, Sugahara H, et al. Age-related changes in gut microbiota composition from newborn to centenarian: a cross-sectional study. BMC Microbiol 2016;16:90.

5. Singh RK, Chang HW, Yan D, et al. Influence of diet on the gut microbiome and implications for human health. J Transl Med 2017;15:73.

6. Tseng CH, Wu CY. The gut microbiome in obesity. J Formos Med Assoc 2019;118 Suppl 1:S3-S9.

7. Shreiner AB, Kao JY, Young VB. The gut microbiome in health and in disease. Curr Opin Gastroenterol 2015;31:69-75.

8. Mallika L, Augustine D, Rao RS, et al. Does microbiome shift play a role in carcinogenesis? A systematic review. Transl Cancer Res 2020;9:3153-66.

9. Liu K, Lu C. Gut microbes modulate host response to immune checkpoint inhibitor cancer immunotherapy. Transl Cancer Res 2018;7:S608-10.

10. Jiang C, Li G, Huang P, et al. The Gut Microbiota and Alzheimer's Disease. J Alzheimers Dis 2017;58:1-15.

11. Castillo-Rodriguez E, Fernandez-Prado R, Esteras R, et al. Impact of Altered Intestinal Microbiota on Chronic Kidney Disease Progression. Toxins (Basel) 2018;10:300.

12. Szeligowski T, Yun AL, Lennox BR, et al. The Gut Microbiome and Schizophrenia: The Current State of the Field and Clinical Applications. Front Psychiatry 2020;11:156.

13. Kazemian N, Mahmoudi M, Halperin F, et al. Gut microbiota and cardiovascular disease: opportunities and challenges. Microbiome 2020;8:36.

14. Tang WHW, Kitai T, Hazen SL. Gut Microbiota in Cardiovascular Health and Disease. Circ Res

Cite this article as: Shivani S, Chattopadhyay A, Chuang EY. Targeting the gut microbiome for non-communicable diseases: present and future. Ann Transl Med 2021;9(5):361. doi: 10.21037/ atm-21-144
2017;120:1183-96.

15. Yin J, Liao SX, He Y, et al. Dysbiosis of Gut Microbiota With Reduced Trimethylamine-N-Oxide Level in Patients With Large-Artery Atherosclerotic Stroke or Transient Ischemic Attack. J Am Heart Assoc 2015;4:e002699.

16. Ramezani A, Raj DS. The Gut Microbiome, Kidney Disease, and Targeted Interventions. JASN 2014;25:657-70.

17. Wilkins LJ, Monga M, Miller AW. Defining Dysbiosis for a Cluster of Chronic Diseases. Sci Rep 2019;9:12918.

18. Shen Y, Xu J, Li Z, et al. Analysis of gut microbiota diversity and auxiliary diagnosis as a biomarker in patients with schizophrenia: A cross-sectional study. Schizophr Res 2018;197:470-7.

19. Zheng P, Zeng B, Liu M, et al. The gut microbiome from patients with schizophrenia modulates the glutamateglutamine-GABA cycle and schizophrenia-relevant behaviors in mice. Sci Adv 2019;5:eaau8317.

20. Jouanne M, Rault S, Voisin-Chiret AS. Tau protein aggregation in Alzheimer's disease: An attractive target for the development of novel therapeutic agents. Eur J Med Chem 2017;139:153-67.

21. Köhler CA, Maes M, Slyepchenko A, et al. The Gut-Brain Axis, Including the Microbiome, Leaky Gut and Bacterial Translocation: Mechanisms and Pathophysiological Role in Alzheimer's Disease. Curr Pharm Des 2016;22:6152-66.

22. Cherny I, Rockah L, Levy-Nissenbaum O, et al. The formation of Escherichia coli curli amyloid fibrils is mediated by prion-like peptide repeats. J Mol Biol 2005;352:245-52.

23. Chen SG, Stribinskis V, Rane MJ, et al. Exposure to the Functional Bacterial Amyloid Protein Curli Enhances Alpha-Synuclein Aggregation in Aged Fischer 344 Rats and Caenorhabditis elegans. Sci Rep 2016;6:34477.

24. Wang X, Sun G, Feng T, et al. Sodium oligomannate therapeutically remodels gut microbiota and suppresses gut bacterial amino acids-shaped neuroinflammation to inhibit Alzheimer's disease progression. Cell Res 2019;29:787-803.

25. Devlin AS, Marcobal A, Dodd D, et al. Modulation of a Circulating Uremic Solute via Rational Genetic Manipulation of the Gut Microbiota. Cell Host Microbe 2016;20:709-15. 